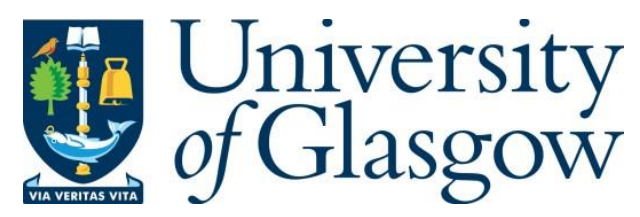

Cha, C. B., O'Connor, R. C., Kirtley, O., Cleare, S., Wetherall, K., Eschle, S., Tezanos, K. M. and Nock, M. K. (2018) Testing mood-activated psychological markers for suicidal ideation. Journal of Abnormal Psychology, 127(5), pp. 448-457.

There may be differences between this version and the published version. You are advised to consult the publisher's version if you wish to cite from it.

http://eprints.gla.ac.uk/160593/

Deposited on: 12 April 2018

Enlighten - Research publications by members of the University of Glasgow http://eprints.gla.ac.uk 
Words: 8,386

Tables: 2

Figures: 1

Running head: MOOD-ACTIVATED MARKERS FOR SUICIDE

\title{
Testing mood-activated psychological markers for suicidal ideation
}

\author{
Christine B. Cha, Ph.D. ${ }^{a}$ \\ Rory C. O’Connor, Ph.D. ${ }^{b}$ \\ Olivia Kirtley, Ph.D., \\ Seonaid Cleare, B.Sc. ${ }^{\text {b }}$ \\ Karen Wetherall, M.Sc. ${ }^{b}$ \\ Sarah Eschle, M.A. ${ }^{\text {b }}$ \\ Katherine M. Tezanos, B.A. ${ }^{a}$ \\ Matthew K. Nock, Ph.D. ${ }^{\text {d }}$
}
${ }^{a}$ Department of Counseling \& Clinical Psychology, Teachers College, Columbia University
${ }^{\mathrm{b}}$ Suicidal Behavior Research Laboratory, Institute of Health \& Wellbeing, University of
Glasgow
${ }^{\mathrm{c}}$ Center for Contextual Psychiatry, Department of Neuroscience, KU Leuven
${ }^{\mathrm{d}}$ Department of Psychology, Harvard University

Correspondence to: Christine B. Cha, Ph.D., Department of Counseling \& Clinical Psychology, Teachers College, Columbia University, 525 West $120^{\text {th }}$ Street, Box 102, New York, NY 10027, e-mail: cbc2120@tc.columbia.edu, phone: +1 (212) 678-8212. 


\begin{abstract}
To what extent are death- and life-oriented psychological processes among suicidal individuals activated by mood? According to Teasdale's (1988) Differential Activation Hypothesis, we would expect that negative mood-activated psychological processes are maladaptive among suicide ideators (vs. non-ideators), and predictive of subsequent suicidal ideation. This, however, has never been prospectively studied. To address this knowledge gap, we conducted a prospective study assessing psychological risk factors via the Death/Life Implicit Association Test (IAT) and the Suicide Stroop task before and after a temporary negative mood induction. Suicidal ideation was assessed one- and six-months later. Results based on Death/Life IAT performance largely supported hypotheses, such that suicide ideators demonstrated significantly weaker implicit identification with life after (vs. before) the negative mood induction. Nonideators demonstrated no significant change, maintaining strong identification with life irrespective of mood. Of note, this baseline interaction may have been accounted for by depressive symptoms. Identification with death (vs. life) predicted greater likelihood of suicidal ideation one month later, controlling for depressive symptoms and baseline suicidal ideation. Only negative mood-activated identification with death predicted suicidal ideation six months later. Suicide Stroop scores did not change as a function of mood or predict subsequent suicidal ideation. Death/Life IAT findings support the Differential Activation Hypothesis and suggest that suicide ideators' identification with life is more variable and easily weakened by negative mood relative to non-ideators. We encourage future work to consider the potential role of transient mood and the importance of measuring psychological processes that pertain to both death and life.
\end{abstract}




\section{General Scientific Summary}

This study examines negative mood-activated psychological processes among suicidal individuals. Using the Death/Life Implicit Association Test (IAT), we found that suicide ideators show weakened identification with life after a negative mood induction relative to non-ideators. This baseline interaction with mood may be accounted for by depressive symptoms. Moodactivated psychological processes, relative to psychological processes observed in a neutral state, are especially predictive of future suicidal ideation even after controlling for depressive symptoms and baseline suicidal ideation. These patterns were not observed for another psychological process pertaining solely to death and suicide - as captured by the Suicide Stroop task. These findings suggest that researchers and clinicians should consider the respondent's transient mood when using the Death/Life IAT as an assessment of suicide risk.

Keywords: Suicidal ideation, Implicit Association Test (IAT), Emotional Stroop, Differential Activation Theory, risk assessment 
Suicidal ideation is a prevalent, harmful, and inherently distressing experience. Approximately $10 \%$ of individuals have had suicidal thoughts at least once in their life (Nock et al., 2008), and suicide ideators are at elevated risk of maladaptive outcomes, including suicide attempt (Reinherz, Tanner, Berger, Beardslee, \& Fitzmaurice, 2006). Suicidal ideation is difficult to predict; as a recent meta-analysis shows, we have not improved our ability to predict suicidal thoughts and behaviors over the past 50 years (Franklin et al., 2017). This finding calls for theoretically- and empirically-informed improvements in how we predict these life-threatening outcomes.

Testing psychological risk factors for suicidal ideation typically involves assessing cognitive or affective processes once at baseline, and measuring the later occurrence of suicidal thoughts (Franklin et al., 2017). This approach is problematic because suicidal ideation can be transient in nature (e.g., Kleiman et al., 2017), and therefore remains unlikely to be predicted by static risk factors. There is a need to determine not only which risk factors predict this clinical outcome, but also when and under what conditions prediction is optimal. The current investigation focuses on the impact of transient negative mood, and whether negative moodactivated psychological risk factors better differentiate suicidal from nonsuicidal individuals.

Prior work suggests that certain psychological risk factors may not distinguish suicidal from nonsuicidal individuals unless they are activated by a negative mood state (Lau, Segal, \& Williams, 2004). The activation of psychological processes is traditionally measured through the use of a mood induction paradigm (e.g., Gemar, Segal, Sagrati, \& Kennedy, 2001; O’Connor \& Williams, 2014; Teasdale, 1988; Williams, Barnhofer, Crane, \& Beck, 2005; Williams, Van der Does, Barnhofer, Crane, \& Segal, 2008). The use of mood induction paradigms helps determine the degree to which maladaptive psychological processes are activated by negative mood and are associated with recurrent depression (Teasdale, 1983). Despite its proposed role as a causal risk 
factor for depression and possibly suicidality (Lau et al., 2004), the potential interactive effects of negative mood are rarely acknowledged or used to inform how we study suicide risk.

The theoretical rationale for measuring mood-activated psychological processes lies in Teasdale's Differential Activation Hypothesis (1988). Originally developed to explain depression vulnerability, the Differential Activation Hypothesis proposes that people vary in how their maladaptive psychological processes respond to negative mood. Some people endorse psychological processes that are more highly activated by negative mood, and they are more likely to be persistently depressed, whereas others have greater resistance to negative mood and are less likely to experience persistent depression (e.g., Segal, Gemar, \& Williams, 1999; Segal, Kennedy, Gemar, Hood, Pedersen, \& Buis, 2006; Werner-Seidler \& Moulds, 2011; Yeung, Dalgleish, Golden, \& Schartau, 2006). Negative mood-activated psychological processes are argued to emerge from 'configurations' of thought patterns and information processing biases that individuals rehearse during initial depressive episodes (Segal, Williams, Teasdale, \& Gemar, 1996). The link between negative mood and its corresponding psychological process is argued to strengthen over time, to a point where the psychological processes can increasingly be primed and activated by negative mood states. Importantly, the Differential Activation Hypothesis can be applied to a wide variety of processes, ranging from autobiographical memory (e.g., Yeung et al., 2006) to dysfunctional attitudes (Segal et al., 2006).

Mood-activated psychological processes may be especially relevant to suicidal ideation. Lau and colleagues (2004) extended the Differential Activation Hypothesis and asserted that those endorsing high activation may be more likely to experience suicidal thoughts and behaviors in the future, given that suicidal ideation often emerges in depressive episodes where maladaptive psychological processes are rehearsed. This maps onto the known transient nature of suicidal thoughts (Kleiman et al., 2017). Indeed, suicidal individuals have been found to 
demonstrate more maladaptive psychological processes (e.g., less effective problem-solving solutions) immediately after a negative mood induction, relative to depressed nonsuicidal individuals (Williams et al., 2005). It still remains possible that some mood activation may be accounted for by depression as a whole, given that suicidal and nonsuicidal depressed individuals demonstrate similar impact of negative mood on other aspects of problem-solving (e.g., generation of relevant problem-solving steps; Williams et al., 2005). Regardless, the potential role of negative mood highlights a key consideration that is often missed in suicide research.

Building on cross-sectional work examining mood activation among suicidal individuals (Williams et al., 2005; Williams et al., 2008), this study represents the first prospective examination of whether negative mood-activated psychological processes predict suicidal ideation over time. This is examined using a number of different maladaptive psychological processes. These may feature either strengthened bias toward negatively-valenced content (e.g., dysfunctional attitudes, memory biases toward negative information; Gilboa \& Gotlib, 1997; Segal et al., 2006), or weakened bias toward positively-valenced content (e.g., positive future thinking, effectiveness of problem-solving solutions; O’Connor \& Williams, 2014; Williams et al., 2008). To test the breadth of mood activation among suicidal individuals, we examined two separate psychological processes_ - one pertaining to death, and the other pertaining to the dichotomy of death and life. These psychological processes, described below, come from distinct parts of the literature and thereby test the generalizability of Teasdale's (1988) Differential Activation Hypothesis.

One psychological process we examined was attentional bias toward death- and suiciderelevant stimuli. According to the Cognitive Model of Suicide (Wenzel \& Beck, 2008), suicidal individuals have a particular difficulty inhibiting suicide-related thought content as a result of an activated suicide schema (i.e., trait hopelessness, unbearability). This form of attentional bias can 
be captured by an adapted version of the emotional Stroop task, commonly referred to as the Suicide Stroop task (Becker, Strohbach, \& Rinck, 1999; Cha, Najmi, Park, Finn, \& Nock, 2010; Williams \& Broadbent, 1986). ${ }^{1}$ Based on the assumption that emotionally salient content interferes with one's ability to attend to other aspects of the word (e.g., font color), the Suicide Stroop task measures reaction times of identifying the color of suicide-related (vs. neutral) words. Earlier studies have demonstrated that interference with suicide-related words is associated with recent suicide attempt (Williams \& Broadbent, 1986), and that this association is specific to suicide-related content since no such interference appears with general negativelyvalenced words (Becker et al., 1999). A more recent study prospectively demonstrated that performance on this task can predict future suicide attempt, even when controlling for wellknown risk factors (e.g., mood disorder, prior suicide attempt) and clinicians' and patients' own prediction (Cha et al., 2010).

We also examined the degree of identification with death, relative to identification with life. This is a psychological process characterized by the automatic tendency to pair concepts of oneself with life more readily than with death. The desire to live is proposed as a powerful barrier to suicide risk: the stronger this barrier, the less likely it is that one will want or try to kill themselves (Joiner, 2005). This implicit identification with death versus life is captured through the Death/Life Implicit Association Test (Death/Life IAT; Nock, Park, Finn, Deliberto, Dour, \& Banaji, 2010). Based on the assumption that people pair related constructs more quickly than unrelated constructs, the Death/Life IAT captures reaction times of endorsing one association

\footnotetext{
${ }^{1}$ We acknowledge that the Suicide Stroop task assesses attentional bias in the context of stimuli not directly having to do with 'suicide' (e.g., dead, funeral). We maintain the name Suicide Stroop task in the present article to maintain consistency with prior papers on this topic, and to reflect the originally intended purpose of this task which was to study suicidal individuals. And while we adhere to the traditional term 'attentional bias' to maintain consistency with prior literature (e.g., Becker et al., 1999; Cha et al., 2010; Chung \& Jeglic, 2016; Williams \& Broadbent, 1986; Williams, Mathews, \& MacLeod, 1996), we acknowledge that the emotional Stroop task has generally been noted for not capturing selective attention, and instead representing cognitive avoidance (e.g., de Ruiter \& Brosschot, 1994) or a generic slow-down effect (e.g., Algom, Chajut, \& Lev, 2004).
} 
(e.g., Death $=M e$ ) versus another (e.g., Life $=M e$; Nock et al., 2010). Whereas most people strongly identify with life (i.e., Life $=M e /$ Death $=$ Not $M e$ ), suicide ideators weakly associate the concept of themselves with life (i.e., Death=Me / Life=Not Me; Glenn et al., 2017; Harrison, Stritzke, Fay, Ellison, \& Hudaib, 2014). A person may not be fully aware of them, but implicit cognitions can guide future decisions and/or behaviors in a way that is critical to their wellbeing. Indeed, Death/Life IAT performance has been shown to predict future suicide attempt above and beyond well-known risk factors (e.g., mood disorder, prior suicide attempt) in addition to clinician and patient prediction as measured via explicit self-report (Nock et al., 2010). This effect is robust and sensitive to frequency of suicide attempts and recency of selfinjury (Glenn et al., 2017).

In the only study to test Death/Life IAT performance after a mood induction, Tang and colleagues (2013) found that individuals identify more weakly with life after a brief, failurerelated priming paradigm, compared to success-related priming paradigm and no-paradigm conditions. This suggests that negative mood in particular may account for weaker identification with life. Despite this promising evidence, several key limitations of the former study remain. First, there was no direct measure of suicidal thoughts and behaviors. It therefore remains unknown whether there is greater weakening of identification with life among suicidal (vs. nonsuicidal) individuals. Second, there was no baseline (i.e., pre-induction) assessment of Death/Life IAT performance, thereby leaving unknown whether there was a significantly weakened identification with life over time. Third, this was a cross-sectional study, limiting conclusions about the prospective validity of these mood-activated effects.

Addressing the aforementioned limitations, the current study tested whether life- and death-oriented psychological processes_ captured by the Suicide Stroop task and Death/Life IAT — are activated by negative mood among suicidal individuals (vs. nonsuicidal individuals), 
and then prospectively tests the predictive validity of activated vs. non-activated biases.

Specifically, the present laboratory-based study explored the effects of a temporary negative mood induction on attentional bias and implicit identification with death versus life. We tested two hypotheses pertaining to whether the biases were not activated (i.e., pre-induction) or activated (i.e., post-induction) by negative mood. First, we hypothesized that suicide ideators would exhibit greater activation of psychological processes (i.e., weaker post-induction bias toward life; stronger post-induction bias toward death), relative to non-ideators. We expected little to no activation of psychological processes among nonsuicidal adults. Second, we hypothesized that activated psychological processes would more strongly predict future suicidal ideation above and beyond key covariates and baseline suicidal ideation.

\section{Method}

These hypotheses were tested via two studies simultaneously conducted across two psychology research laboratories, one in Cambridge, Massachusetts (Site 1) and the other in Central Scotland (Site 2). Research teams at each site initially shared training and study materials and then recruited and conducted the studies independently. Methodological approaches were identical, and sites were comparable across key variables; ${ }^{2}$ their Methods and Results are therefore presented together. Supplemental materials feature site-level results.

\section{Sample}

\footnotetext{
${ }^{2}$ These two sites were comparable across key variables (Supplemental Table 1). First, there were no cross-site differences in key variables such as Death/Life IAT scores and Stroop task scores. Second, the sites did not demonstrate differences in primary outcomes (i.e., proportions of baseline, 1-month, and 6-month suicidal ideation. Of note, sites demonstrated differing qualities of suicidal ideation. This was especially the case at baseline, where Site 1 suicide ideators reported experiencing more frequent, yet less intense and shorter-lasting, instances of suicidal ideation compared to Site 2 suicide ideators. The only difference in follow-up suicidal ideation was at the 1-month follow-up period, where Site 1 suicide ideators reported experiencing more frequent (yet similar in duration) instances of suicidal ideation. There were no site differences in quality of suicidal ideation at the 6-month follow-up time point.
} 
Participants were 264 suicidal and nonsuicidal adults from the greater Boston area $(n=143)$ and from Central Scotland (Stirling and Glasgow; $n=121)$. Across both sites, research teams recruited community-based adults through online postings (e.g., Craigslist, Gumtree), as well as flyers and newspaper ads. Potential participants completed a short screening phone call, and adults were considered suicide ideators $(n=176,66.7 \%)$ if they reported past year ${ }^{3}$ history of suicidal ideation (i.e., in response to Have you ever actually had thoughts about killing yourself? If so, when was the last time?), and again endorsed suicidal ideation when completing the SelfInjurious Thoughts and Behaviors Interview (SITBI; Nock et al., 2007) during the laboratory visit. Adults were considered non-ideators $(n=88,33.3 \%)$ if they reported no lifetime history of suicidal ideation or attempt and a high current desire to live (i.e., an answer of ' 10 ' in response to the question, Currently, how would you rate your desire to live, with '10' being that you really want to be alive, and ' 0 ' that you very much want to be dead?) during the phone screen. This multi-item approach to identifying suicide ideators and non-ideators helps to address potential misclassification errors that can occur when using single-item assessments of suicidal thoughts and behaviors (Millner, Lee, \& Nock, 2015). Inclusion criteria were: adult status ( $\geq 18$ years-old), English fluency, and availability to complete the full two-hour in-person interview and two follow-up telephone interviews. We did not recruit individuals who indicated the presence of any factor impairing the individual's ability to effectively participate in the study, including the presence of gross cognitive impairment, agitated/violent behavior, or high/imminent risk of suicide. Site-specific details comparing key measures and sample characteristics are described below.

\footnotetext{
${ }^{3}$ A total of 16 individuals recruited to the study were excluded, as they had either reported experiencing suicidal ideation in their lifetime but not in the past year during the phone screen $(n=7)$, or endorsed past-year suicidal ideation during the phone screen but not during the SITBI $(n=8)$. In addition, there was one participant who withdrew from the study during baseline assessment, resulting in a final sample of 264 . There were three participants whose phone screen data were not recorded, in which case SITBI responses about past year suicidal ideation were used instead.
} 
Among the 264 individuals from baseline assessment, 228 (86.4\%) completed the 1month follow-up assessment, and $214(81.1 \%)$ completed the 6-month follow-up assessment. Within the 1-month follow-up sample, 64 individuals (28.1\%) endorsed suicidal ideation. Within the 6-month follow-up sample, 90 (42.1\%) endorsed suicidal ideation.

\section{Measures}

Death/Life Implicit Association Test (IAT). We used the Death/Life IAT (Nock et al., 2010) to measure implicit identification with death versus life. The Death/Life IAT is a brief reaction time test during which participants sort words into concept categories (Death, Life) and attribute categories $(\mathrm{Me}, \mathrm{Not} \mathrm{Me})$ by pressing either a left or right key on a computer keyboard. Half of the trials presented category pairs of Death/Me on one side and Life/Not Me on the other side; the other half presented category pairs of Death/Not Me on one side and Life/Me on the other side. These were the only concept pairings featured in the task. The Death/Life IAT compares the speed at which a person classifies stimuli when the paired concept-attribute categories match an individual's implicit associations (e.g., Life/Me), versus the speed of classification when the paired categories do not match an individual's implicit associations (e.g., Life/Not Me). Shorter response latencies indicate stronger implicit associations between the paired concept-attribute categories. In this case, we would expect those with a history of suicidal ideation to categorize life-related words into the Life category slower when Life is paired with $M e$ (vs. Not Me), and death-related words into the Death category faster when Death is paired with $M e$ (vs. Not Me). This is indicated by a higher and less negative IAT $D$ score, whose calculation is based on prior IAT work (e.g., Greenwald, Nosek, \& Banaji, 2003). See Nock et al. (2010) for a more detailed description.

Suicide Stroop task. We used a Suicide Stroop task (Becker et al., 1999; Cha et al., 2010; Williams \& Broadbent, 1986) to measure attentional bias. The Suicide Stroop task is a 
computerized, adapted emotional Stroop task which presents a series of words on the screen that are printed in either red or blue. Participants identified the color of the word by pressing one of two different keys. Most relevant to the current investigation, among the different words participants saw, some were death- and suicide-related words (dead, funeral, suicide) and some were neutral words (paper, engine, museum). See Cha et al. (2010) for measure details. We measured response latencies from identifying font color: greater response latencies were interpreted as indicating greater interference due to the semantic content of the presented words. For example, we would expect an individual with attentional bias to demonstrate greater response latencies during suicide-related word trials.

Negative mood induction. We used a Velten mood induction (Velten, 1968) to temporarily induce negative mood among participants prior to retaking the Death/Life IAT and Suicide Stroop task. This 10-minute mood induction paradigm consisted of participants reading negative statements and being asked to reflect on how those statements apply to them (e.g., There are things about me that I do not like; The way I feel now, the future looks boring and hopeless). While reading these statements, participants also listened to "Russia under the Mongolian Yoke" by Prokofiev played at half speed. This procedure is identical to the one used in prior studies involving adult suicide ideators (Williams et al., 2005, 2008) and in studies producing evidence for mood-activated psychological processes in depression (see Clark, Beck, \& Alford, 1999).

Transient mood assessment. Transient mood was assessed using a two-dimensional visual analog scale (VAS) aligned with the Circumplex Model of emotions (Russell, 1980). This is adapted from Russell and colleagues' (1989) Affect Grid, which is a single-item scale indicating degree of valence along the horizontal axis (negative-positive) and arousal along the vertical axis (high-low). Participants were asked to mark one coordinate indicating both valence 
and arousal in that moment. Mood valence was measured by the horizontal coordinate such that higher values indicated more positive mood and lower values indicated more negative mood. Arousal was measured by the vertical coordinate such that higher values indicated higher arousal, and lower values indicated lower arousal.

Assessment of suicidal ideation. Suicidal ideation was assessed during the phone screen prior to the lab visit and through the Self-Injurious Thoughts and Behaviors Interview (SITBI; Nock et al., 2007) during the lab visit. Response to the suicidal ideation questions in the SITBI (i.e., Have you ever had thoughts of killing yourself?) was used to corroborate phone screenbased reports of past-year suicidal ideation history. The SITBI was also used to assess suicidal ideation one- and six-months after the laboratory visit. At each time point, presence (vs. absence) of suicidal ideation within a specific time frame (at baseline - past year; 1-month follow-uppast 1 month; 6-month follow-up_-past 6 months) was treated as the outcome variable. Participants also estimated the number of days (i.e., frequency) they had experienced suicidal ideation over the past year; this was included as a covariate in prospective analyses.

Assessment of depressive symptoms. We administered the Beck Depression Inventory (BDI-II; Beck, Steer, \& Brown, 1996) to assess depressive symptoms experienced over the past two weeks. The total score was calculated excluding the item about suicidal ideation.

\section{Procedure}

In the laboratory, participants completed an assessment of their baseline mood, along with the pre-induction Death/Life IAT and Stroop task. Behavioral tasks were presented in random order, and there were no order effects between groups or on task performance, $p \mathrm{~s}=.21$ 98. Participants next completed the negative mood induction, immediately followed by the postinduction Death/Life IAT and Stroop task administered in the same order as the pre-induction tasks. They then completed the SITBI, a clinical interview, and self-report measures. Transient 
mood was measured throughout the lab visit via valence and arousal ratings. At the end of the visit participants completed a 10-minute positive mood induction (e.g., Candid Camera clips).

Finally, participants were contacted by phone one- and six-months later, and were asked questions to assess whether they had engaged in subsequent suicidal thoughts or behaviors. This was measured by participants' responses to a modified version of the SITBI (Nock et al., 2007). Participants were compensated with cash for completing baseline assessment $(\$ 20$ in the greater Boston area and $£ 20$ in Central Scotland) and a gift card ( $\$ 10$ in the greater Boston area and $£ 10$ in Central Scotland) each time they completed a follow-up assessment. Studies were approved by the respective Institutional Review Board of each site (Harvard FAS IRB \#F-22117; Glasgow IRB \#200130010).

\section{Data Analysis}

We conducted independent samples $t$ tests, correlations, and chi-square analyses to test for potential baseline associations with ideation status and task performance (e.g., demographic characteristics, depressive symptoms). We also conducted a manipulation check via paired and independent sample $t$ tests to determine whether the negative mood induction had its intended effect at each site. Participants who produced valid pre- and post-induction scores on the IAT and Suicide Stroop task, and who completed the SITBI question about ideation status, were included in all subsequent analyses. ${ }^{4}$

To test the first hypothesis, we conducted repeated measures ANCOVAs with Suicide Stroop and Death/Life IAT scores testing for Time (pre vs. post) by Group (ideator vs. nonideator) interactions, controlling for multiple covariates identified via preliminary analyses

\footnotetext{
${ }^{4}$ Across sites, 3.8-4.5\% of participants did not produce valid IAT data (due to e.g., errors, overly slow/fast reaction times - see Nock et al., 2010 for scoring approach), and 8.0-10.2\% of participants did not produce valid Stroop data (due to the same reasons as IAT - see Cha et al., 2010 for scoring approach) and were handled via pairwise deletion. Little's (1988) Missing Completely at Random test indicated non-significant results, suggesting that these data were missing at random.
} 
(described below). We also examined whether task scores at each time point (pre- vs. postinduction) differentiated baseline suicide ideators from non-ideators controlling for the aforementioned covariates. These analyses were also conducted with dichotomous scores, which help assess whether any participant not only experienced stronger or weaker identification with life, but instead crossed the threshold to strongly identify with death. Specifically, a dichotomous 'positive' or 'negative' score could be determined based on whether the continuous Death/Life IAT score was a numerical value above or below zero. An IAT $D$ score above zero (i.e., positive) on the Death/Life IAT indicates that the participant responded more quickly in IAT trials where Death and $M e$ were presented together; an IAT $D$ score below zero (i.e., negative) means that the participant responded more quickly in IAT trials where Life and Me were presented together. ${ }^{5}$ This is becoming an increasingly adopted scoring system; it is more easily interpretable (e.g., Cha et al., 2016; Nock et al., 2010) and has been shown to predict suicide attempt (Nock et al., 2010).

Prospective analyses addressing the second hypothesis were conducted to predict suicidal ideation 1- and 6-months later. Suicidal ideation was treated as a dichotomous outcome (i.e., presence vs. absence during the follow-up period). We tested this hypothesis via bivariate and multivariate logistic regression models, with multivariate models controlling for all aforementioned covariates, as well as baseline suicidal ideation (i.e., past year frequency of suicidal ideation).

\section{Results}

\section{Preliminary Analyses}

\footnotetext{
${ }^{5}$ Similarly, a positive interference score on the Suicide Stroop task means that the participant experienced more interference with suicide-related words relative to neutral words; a negative interference score means that the participant experienced more interference with neutral words relative to suicide-related words.
} 
We examined whether baseline characteristics differentiated groups (suicide ideators vs. non-ideators), or were associated with IAT and Stroop performance. Regarding group differences, baseline suicide ideators were slightly younger $(M=31.44, S D=12.14)$ than nonideators, $(M=35.58, S D=15.89), t=2.15, p=.03, d=0.36$. Moreover, a greater proportion of male (73.5\%; vs. female, $61.2 \%$ ) participants were likely to endorse suicidal ideation, $\chi^{2}=4.42, p=.04$, $\Phi=0.13$. Suicide ideators, as expected, endorsed more depressive symptoms than did nonideators, $t=-17.99, p<.001, d=2.23$.

Regarding associations with IAT and Stroop performance (Table 1), the only demographic characteristic associated with task performance was age, such that older participants demonstrated lower pre- and post-induction IAT scores. In contrast, older participants demonstrated higher pre-induction Stroop interference scores. Both sets of IAT scores were associated with depressive symptoms and mood valence, such that more depressive symptoms and pre-/post-induction negative mood valence were associated with higher IAT scores. Stroop scores were neither associated with depressive symptoms nor with mood valence. Neither IAT nor Stroop performance was associated with self-reported arousal.

Manipulation checks revealed that the negative mood induction had its intended effect. ${ }^{6}$ There was a large and significant change from positive to negative mood valence at both sites, $p s<.001, d s=0.60-0.95$. Neither site observed a significant change in arousal, $p s=.19-.82$, $d s=0.02-0.16$. Of note, suicide ideators rated themselves as having significantly more negative mood than non-ideators both before and after the mood induction, $p \mathrm{~s}=.00-.01, d \mathrm{~s}=.50-1.48$. Ideators and non-ideators did not differ in arousal levels before and after the mood induction. $p \mathrm{~s}=.07-.32, \mathrm{ds}=-.18-.31$.

${ }^{6}$ To counteract the effects of the negative mood induction, participants also completed a positive mood induction at the end. Ultimately, participants at both sites reported overall improvement in mood across the lab visit, and reported more positive mood at the end than at the beginning, $p \mathrm{~s}<.001, d \mathrm{~s}=0.41-0.45$. They also reported higher arousal at the end than at the beginning of the lab visit, $p s<.001, d s=0.31-0.44$. 
In subsequent analyses, we controlled for a number of demographic, clinical, and contextual factors based on preliminary findings. Specifically, we controlled for age, gender, depressive symptoms, and pre-/post-induction mood valence given their associations with baseline ideation status and with some task performance. Site was also controlled for in subsequent analyses.

\section{Does negative mood activate psychological processes among baseline suicide ideators, relative to non-ideators?}

Negative mood weakened implicit identification with life among suicide ideators, relative to non-ideators. Suicide ideators had higher IAT scores than non-ideators, $F(1,251)=27.24$, $p<.001, \eta_{p}^{2}=.10$, and this difference was more pronounced after the negative mood induction, as indicated by a significant Group x Time interaction, $F(1,251)=4.39, p=.04, \eta_{p}^{2}=.02$ (Figure 1a). Indeed, suicide ideators had higher IAT scores compared to non-ideators at both time points, with a larger between-group effect after the mood induction, $d=0.83$, compared to before, $d=0.53$. There was a significant main effect of Time, $F(1,251)=5.64, p=.02, \eta_{p}{ }^{2}=.02$, but specifically with IAT scores increasing among ideators, $t(169)=3.78, p<.001, d=0.29$, and remaining unchanged among non-ideators, $t(82)=0.19, p=0.85, d=0.02$. Each site revealed the same directionality of effects, such that suicide ideators' implicit identification with life appeared weaker after the negative mood induction compared to baseline, and appeared relatively unchanged among nonideators (Supplemental Figure 1).

The Group x Time interaction was no longer significant after controlling for relevant demographic, clinical, and contextual correlates (i.e., site, age, gender depressive symptoms, preand post-induction mood valence), $F(1,241)=2.00, p=.16, \eta_{p}^{2}=.01$, suggesting that these factors 
may account for observed pre-/post-induction changes among ideators. It was specifically when depressive symptoms were added as a covariate that the interaction became nonsignificant, pointing to the potentially salient role of depression. Of note, however, results were more promising when each time point was assessed individually. Accounting for the aforementioned covariates, post-induction IAT scores remained significantly associated with suicidal ideation status, $O R=5.00, C I=1.53-16.36, p=.01$, whereas pre-induction IAT scores did not, $O R=1.48$, $C I=0.43-5.06, p=.53$. Results were identical when Site 1 was assessed separately. When Site 2 was assessed separately, neither pre- nor post-induction scores significantly differentiated groups controlling for the aforementioned covariates. Whereas the post-induction dichotomous IAT scores produced larger effect sizes than pre-induction dichotomous IAT scores, neither were significantly associated with suicidal ideation status after accounting for the aforementioned covariates, $O R \mathrm{~s}=1.17-2.50, C I \mathrm{~s}=0.23-9.75, p \mathrm{~s}=.19-.78$. Results involving dichotomous IAT scores were identical across Sites 1 and 2.

In the case of attentional bias, there was no significant main effect of Group or Time,

$F \mathrm{~s}=0.17-0.98, p \mathrm{~s}=.32-.68, \eta_{p}^{2}=.00$, and no significant Group $\mathrm{X}$ Time interaction, $F(1,226)=0.13$, $p=.72, \eta_{p}{ }^{2}=.00$. Neither the continuous Stroop scores (pre/post) nor the dichotomous scores (pre/post) differentiated ideators from non-ideators, $O R \mathrm{~s}=1.10-1.25, C I \mathrm{~s}=0.66-2.12, p \mathrm{~s}=.41-.66$. Results were identical at across Sites 1 and 2.

\section{Do negative mood-activated psychological processes predict future suicidal ideation?}

Post-induction IAT scores predicted suicidal ideation above and beyond baseline demographic, clinical, and contextual factors, in addition to past year frequency of suicidal ideation (Table 2). This was the case for IAT dichotomous scores, which predicted one-month suicidal ideation based on pre- and post-induction performance, and six-month suicidal ideation 
based on post-induction performance. In other words, those select cases where participants definitely identified more with death (i.e., positive IAT score) than life (i.e., negative IAT score) were more likely to be marked by recurrent suicidal ideation. At the individual site level, bivariate analyses were identical for Site 1 and mostly nonsignificant at Site 2 (Supplemental Table 2). Multivariate analyses were not reported at the site level due to detection of suppressor effects.

Attentional bias did not prospectively predict suicidal ideation at either follow-up time point. No bivariate analyses involving Stroop interference scores significantly predicted followup suicidal ideation, $O R \mathrm{~s}=1.00-1.38, C I \mathrm{~s}=0.55-2.56, p \mathrm{~s}=.09-.97$. Identical findings emerged within each site. Multivariate analyses involving Stroop scores were not conducted.

\section{Discussion}

Three key findings emerged from the current study. First, implicit identification with life weakened after a negative mood induction among suicide ideators, and remained unchanged among non-ideators. Depressive symptoms may account for this interaction, though they do not account for between-group differences observed after the negative mood induction. Second, implicit identification with death (versus life) predicted future suicidal ideation above and depressive symptoms and other key demographic, clinical, and contextual factors. Third, attentional bias did not relate to suicidal ideation, nor was it affected by the negative mood induction. We elaborate on each key finding below.

Our primary finding was that implicit identification with life weakens among suicide ideators (vs. non-ideators) in the context of negative mood, which serves as additional evidence in support of the Differential Activation Hypothesis. This result is consistent with prior crosssectional work examining mood activation among suicidal individuals (Williams et al., 2005, 2008) and using an adapted IAT (Gemar et al., 2001). It shows that, similar to interpersonal 
problem-solving and positive future fluency (Williams et al., 2005, 2008), implicit identification with life is another psychological process that can be activated and specifically weakened by negative mood. Importantly, our results do not suggest that mood-activated psychological processes are especially pronounced or distinct among suicide ideators compared to depressed individuals. Since the Group by Time interaction was not significant, especially once controlling for depressive symptoms, it is likely that the observed mood activation may otherwise be accounted for by depression. This does not necessarily conflict with the Differential Activation Hypothesis, as much of this framework pertains to depressive symptoms. The present investigation simply shows that the suicidal ideation is not an exception to the phenomenon of mood-activated psychological processes.

We also found that nearly all scores (i.e., pre- and post-induction) representing implicit identification with death (vs. life) predicted follow-up suicidal ideation. Findings were especially robust for the Death/Life IAT dichotomous scores (vs. continuous scores), which consistently remained significant when controlling for demographic, clinical, and contextual factors, along with baseline suicidal ideation. This finding highlights the potential importance of the dichotomy between life and death, reinforcing the idea that there is a known tension between the two (Kovacs \& Beck, 1977). On a practical note, the incremental predictive validity of the postinduction Death/Life IAT points to potential ways of optimizing suicide risk assessment toolsespecially when they are administered in a relatively neutral setting.

Contrary to our hypotheses, attentional bias as measured by the Suicide Stroop task did not relate to suicidal ideation at any time point, nor did it seem to change as a function of the negative mood induction. There are several reasons why this may be the case. First, psychological processes toward death such as this may be detectable only among clinically severe populations. The present sample featured suicide ideators from the community, some of 
whom may have never been hospitalized or attempted suicide. This may help explain the lack of significant Stroop findings in the present study and in Chung and Jeglic (2016), versus significant findings as detected in studies taking place in the emergency room (Cha et al., 2010) and inpatient unit/psychiatric ward (Becker et al., 1999). Second, one measure of suicide risk may be less reliable than another measure. Specifically, Suicide Stroop scores have very recently been shown to have unacceptably low internal inconsistency (Wilson et al., 2018). Their clinical utility and ability to track psychological processes over time (pre- vs. post-induction) is likely to be extremely limited. In contrast, the Death/Life IAT has been shown to have very high internal consistency (Glenn et al., 2017), and the IAT has generally demonstrated good test-rest reliability (e.g., Greenwald, \& Farnham, 2000). An improved or alternative measure of psychological processes about death, rather than the Suicide Stroop Task in its present form, may offer a better test of mood activation in future studies. Third, psychological processes toward death may genuinely be less mood-activated than psychological processes involving a dichotomy between death and life. This is consistent with the notion that life-oriented processes (i.e., selfpreservation drives) represent a barrier to suicide risk, such that death-oriented processes can only increase once the basic instinct toward self-preservation has been weakened (Joiner, 2005). Indeed, other psychological processes specifically with a positive (vs. negative) valence have been shown to confer risk of suicidal thoughts and behaviors (e.g., O'Connor, Whyte, Fraser, Masterton, Miles, \& MacHale, 2007; Williams, Ellis, Tyers, Healy, Rose, \& MacLeod, 1996). The durability and temporal association between life- and death-oriented psychological processes thereby represents an area for further study.

Results should be interpreted in light of several limitations. First and foremost, we did not include a control mood manipulation, thereby limiting our interpretation of results. By randomly assigning half of the suicide ideators to receive a sham or neutral mood induction rather than a 
negative mood induction, we could more precisely attribute changes in psychological process to feeling negative or sad and/or rule out potential practice effects. Mood induction studies are encouraged to do so in the future. Second, whereas mood ratings distinguished emotional valence from arousal following Russell's (1980) Circumplex Model of emotions, both sets of ratings still relied on self-report. Self-report measures were used out of feasibility and convenience, but the cost of this assessment method is potentially inaccurate self-report (Tourangeau \& Yan, 2007). Mood inductions absent of demand characteristics (e.g., unsolvable anagram tasks, manipulated achievement tests; Chartier \& Ranieri, 1989; Nock \& Mendes, 2008) offer an alternative mood induction procedure. Applying alternative mood inductions would help tease apart mechanisms driving the observed mood decline. Given the established relationship between stress and suicide risk, it would also be of interest to investigate the extent to which stress-manipulations and the stress response affect psychological processes (O’Connor \& Nock, 2014; O’Connor et al., 2016). Third, because this was a community-based sample, it remains unclear to what extent these findings generalize to a clinical population. And while outside the scope of the current investigation, there was only one suicide attempt reported during the followup periods, limiting our ability to test whether the Death/Life IAT prospectively predicts suicide attempt. This type of finding would have been even further aligned with existing work which used the same version of the IAT to predict suicide attempt rather than suicidal ideation (Nock et al., 2010). A final caveat is our limited sample size. Despite the accumulation of two samples, we remained limited in our ability to test and thereby interpret multivariate effects. Testing larger samples would allow us to further explore this possibility and more reliably test the Differential Activation Hypothesis.

In spite of these limitations, this study extends our conceptual understanding of psychological processes and informs practical administration of newer suicide risk assessments. 
The present findings have a number of clinical implications and highlight the importance of not only which risk factors are assessed, but also when. Both researchers and clinicians are encouraged to consider whether individuals are in a negative mood state, which would offer an opportunity to detect risk factors in a way that is not possible when patients are in a neutral mood state. This informs us of how behavioral measures of suicide risk may be optimally implemented when administered outside of an acute care setting (e.g., primary care setting). An additional clinical implication is the demonstration that these behavioral measures can produce scores that are easily interpretable and require minimum training. This complements the field's current efforts toward generating brief, user-friendly screening instruments (e.g., Horowitz, Ballard, \& Pao, 2009). Continuation of this research will inform thoughtful and impactful application of behavioral measures and ultimately serve a population in need of greater attention. 


\section{REFERENCES}

Algom, D., Chajut, E., \& Lev, S. (2004). A rational look at the emotional Stroop phenomenon: A generic slowdown, not a Stroop effect. Journal of Experimental Psychology: General, $133,323-338$.

Beck, A.T., Steer, R.A., \& Brown, G.K. (1996). Manual for the Beck Depression Inventory-II. San Antonio, TX: Psychological Corporation.

Beck, A.T., Steer, R.A., \& Garbin, M.G. (1988). Psychometric properties of the depression inventory: Twenty-five years of evaluation. Clinical Psychology Review, 8, 77-100.

Becker, E.S., Strohbach, D., \& Rinck, M. (1999). A specific attentional bias in suicide attempters. Journal of Nervous and Mental Disease, 187, 730-735.

Borges, G., Angst, J., Nock, M.K., Ruscio, A.M., \& Kessler, R.C. (2008). Risk factors for the incidence and persistence of suicide-related outcomes: A 10-year follow-up study using the National Comorbidity Surveys. Journal of Affective Disorders, 105, 25-33.

Cha, C.B., Augenstein, T.M., Frost, K.H., Gallagher, K., D’Angelo, E.J., \& Nock, M.K. (2016). Using implicit and explicit measures to predict nonsuicidal self-injury among adolescent inpatients. Journal of the American Academy of Child and Adolescent Psychiatry, 55, 6268.

Cha, C.B., Najmi, S., Park, J.M., Finn, C.T., \& Nock, M.K. (2010). Attentional bias toward suicide-related stimuli predicts suicidal behavior. Journal of Abnormal Psychology, 119, 616-622.

Chartier, G.M., \& Ranieri, D.J. (1989). Comparison of two mood induction procedures. Cognitive Therapy and Research, 13, 275-282.

Chung, Y., \& Jeglic, E.L. (2016). Use of the modified emotional Stroop task to detect suicidality in college population. Suicide and Life-Threatening Behavior, 46, 55-66. 
Clark, D.A., Beck, A.T., \& Alford, B.A. (1999). Scientific foundations of cognitive theory and therapy for depression. New York: John Wiley \& Sons.

De Ruiter, C., \& Brosschot, J.F. (1994). The emotional Stroop interference effect in anxiety: Attentional bias or cognitive avoidance? Behavior Research and Therapy, 32, 315-319.

Franklin, J.C., Ribeiro, J.D., Fox, K.R., Bentley, K.H., Kleiman, E.M., Huwang, X., ...Nock, M.K. (2017). Risk factors for suicidal thoughts and behaviors: A meta-analysis of 50 years of research. Psychological Bulletin, 143, 187-232.

Gemar, M.C., Segal, Z.V., Sagrati, S., \& Kennedy, S.J. (2001). Mood-induced changes on the implicit association test in recovered depressed patients. Journal of Abnormal Psychology, 110, 282-289.

Gilboa, E., \& Gotlib, I.H. (1997). Psychological processes and affect persistence in previously dysphoric and never-dysphoric individuals. Cognitive and Emotion, 11, 517-538.

Glenn, C.R., \& Klonsky, E.D. (2011). Prospective prediction of nonsuicidal self-injury: A 1-year longitudinal study in young adults. Behavior Therapy, 42, 751-762.

Glenn, J.J., Werntz, A.J., Slama, S.J.K., Steinman, S.A., Teachman, B.A., \& Nock, M.K. (2017). Suicide and self-injury-related implicit cognition: A large-scale examination and replication. Journal of Abnormal Psychology, 126, 199-211.

Gotlib, I.H., \& Cane, D.B. (1987). Construct accessibility and clinical depression: A longitudinal investigation. Journal of Abnormal Psychology, 96, 199-204.

Greenwald A.G., Nosek B.A., \& Banaji M.R. (2003). Understanding and using the Implicit Association Test I: An improved scoring algorithm. Journal of Personality and Social Psychology, 85, 197-216.

Greenwald, A.G., \& Farnham, S.D. (2000). Using the Implicit Association Test to measure selfesteem and self-concept. Journal of Personality and Social Psychology, 79, 1022-1038. 
Harrison, D.P., Stritzke, W.G.K., Fay, N., Ellison, T.M., \& Hudaib, A. (2014). Probing the implicit suicidal mind: Does the death/suicide implicit association test reveal a desire to die, or a diminished desire to live? Psychological Assessment, 26, 831-840.

Horowitz, L.M., Ballard, E.D., \& Pao, M. (2009). Suicide screening in schools, primary care and emergency departments. Current Opinion in Pediatrics, 21, 620-627.

Joiner, T.E. (2005). Why people die by suicide. Cambridge, MA: Harvard University Press.

Kleiman, E.M., Turner, B.J., Fedor, S., Beale, E.E., Huffman, J.C., \& Knock, M.K. (2017). Examinations of real-time fluctuations in suicidal ideation and its factors: Results from two ecological momentary assessment studies. Journal of Abnormal Psychology, 126, 726-738.

Kovacs, M., \& Beck, A.T. (1977). The wish to die and the wish to live in attempted suicides. Journal of Clinical Psychology, 33, 361-365.

Lau, M.A., Segal, Z.V., \& Williams, J.M.G. (2004). Teasdale’s Differential Activation Hypothesis: Implications for mechanisms of depressive relapse and suicidal behavior. Behavior Research and Therapy, 42, 1001-1017.

Little, R.J.A. (1988). A test of missing completely at random for multivariate data with missing values. Journal of the American Statistical Association, 83, 1198-1202.

Millner, A.J., Lee, M.D., \& Nock, M.K. (2015). Single-item measurement of suicidal behaviors: Validity and consequences of misclassification. PLoS One, 10, e0141606.

Nock, M.K., \& Cha, C.B. (2009). Psychological models of nonsuicidal self-injury. In M.K. Nock (Ed.), Understanding nonsuicidal self-injury: Origins, assessment, and treatment (pp. 6578). Washington, DC: American Psychological Association 
Nock, M.K., Holmberg, E.B., Photos, V.I., \& Michel, B.D. (2007). The Self-Injurious Thoughts and Behaviors Interview: Development, reliability, and validity in an adolescent sample. Psychological Assessment, 19, 309-317.

Nock, M.K., \& Mendes, W.B. (2008). Physiological arousal, distress tolerance, and social problem solving deficits among adolescent self-injurers. Journal of Consulting and Clinical Psychology, 76, 28-38.

Nock, M.K., Park, J.M., Finn, C.T., Deliberto, T.L., Dour, H., J., \& Banaji, M.R. (2010). Measuring the "suicidal mind:" Implicit cognition predicts suicidal behavior. Psychological Science, 21, 511-517.

O'Connor, D.B., Ferguson, E., Green, J., O'Carroll, R.E., \& O'Connor, R.C. (2016). Cortisol levels and suicidal behavior: A meta-analysis. Psychoneuroendocrinology, 63, 370-379.

O'Connor, R.C., Nock, M.K. (2014). The Psychology of Suicidal Behavior. Lancet Psychiatry, $1,73-85$.

O’Connor, R.C., Whyte, M., Fraser, L., Masterton, G., Miles, J., \& MacHale, S. (2007). Predicting short-term outcome in well-being following suicidal behavior: The conjoint effects of social perfectionism and positive future thinking. Behaviour Research and Therapy, 45, 1543-1555.

O'Connor, R.C., \& Williams, J.M.G. (2014). The relationship between positive future thinking, brooding, defeat and entrapment. Personality and Individual Differences, 70, 29-34

Reinherz, H.Z., Tanner, J.L., Berger, S.R., Beardslee, W.R., \& Fitzmaurice, G.M. (2006). Adolescent suicidal ideation as predictive of psychopathology, suicidal behavior, and compromised functioning at age 30. American Journal of Psychiatry, 163, 1226-1232.

Russell, J.A. (1980). A circumplex model of affect. Journal of Personality and Social Psychology, 39, 1161-1178. 
Russell, J.A., Weiss, A., \& Mendelsohn, G.A. (1989). Affect Grid: A single-item scale of pleasure and arousal. Journal of Personality and Social Psychology, 57, 493-502.

Segal, Z.V., Gemar, M., \& Williams, S. (1999). Differential cognitive response to a mood challenge following successful cognitive therapy or pharmacotherapy of unipolar depression. Journal of Abnormal Psychology, 108, 3-10.

Segal Z. V., Williams J. M. G., Teasdale J. D., \& Gemar M. (1996). A cognitive science perspective on kindling and episode sensitization in recurrent affective disorder. Psychological Medicine, 26, 371-380.

Segal, Z.V., Kennedy, S., Gemar, G., Hood, K., Pedersen, R., \& Buis, T. (2006). Cognitive reactivity to sad mood provocation and the prediction of depression relapse. JAMA Psychiatry, 63, 749-755.

Tang, J., Wu, S., \& Miao, D. (2013). Experimental test of Escape Theory: Accessibility to implicit suicidal mind. Suicide and Life-Threatening Behavior, 43, 347-355.

Teasdale, J.D. (1988). Cognitive vulnerability to persistent depression. Cognition and Emotion, 2, 247-274.

Tourangeau, R., \& Yan, T. (2007). Sensitive questions in surveys. Psychological Bulletin, 133, 859-883.

Velten, E. (1968). A laboratory task for the induction of mood states. Behavior Research and Therapy, 6, 473-482.

Wenzel, A., \& Beck, A.T. (2008). A cognitive model of suicidal behavior: Theory and treatment. Applied and Preventive Psychology, 12, 189-201.

Werner-Seidler, A., \& Moulds, M.L. (2012). Mood repair and processing mode in depression. Emotion, 12, 470-478.

Williams, J.M.G., Barnhofer, T., Crane, C., \& Beck, A.T. (2005). Problem solving deteriorates 
following mood challenge in formerly depressed patients with a history of suicidal ideation. Journal of Abnormal Psychology, 114, 421-431.

Williams, J.M.G., \& Broadbent, K. (1986). Distraction by emotional stimuli: Use of a Stroop task with suicide attempters. British Journal of Clinical Psychology, 25, 101-110.

Williams, J.M.G., Ellis, N.C., Tyers, C., Healy, H., Rose, G., \& MacLeod, A.K. (1996). The specificity of autobiographical memory and imageability of the future. Memory \& Cognition, 24, 116-125.

Williams, J.M.G., Van der Does, A.J.W., Barnhofer, T., Crane, C., \& Segal, Z.S. (2008). Cognitive reactivity, suicidal ideation, and future fluency: Preliminary investigation of a Differential Activation Theory of Hopelessness/Suicidality. Cognitive Therapy and Research, 32, 83-104.

Wilson, K.M., Millner, A.J., Auerbach, R.P., Glenn, C.R., Kearns, J.C., O’Connor, R.C., Kirtley, O., \& Cha, C.B. (2018). Are behavioral measures of suicide risk reliable and valid? An investigation of the Suicide Stroop Task. Manuscript in Preparation.

World Health Organization. (2014). Preventing Suicide: A Global Imperative. WHO, Geneva.

Yeung, C.A., Dalgleish, T., Golden, A.M., \& Schartau, P. (2006). Reduced specificity of autobiographical memories following a negative mood induction. Behavior Research and Therapy, 44, 1481-1490. 


\section{Acknowledgments}

C.B.C. and M.K.N. developed the study concept alongside R.C.O. and O.J.K. Testing and data collection were performed by C.B.C, O.J.K., S.C., K.W., and S.E. under the supervision of M.K.N. and R.C.O. C.B.C. and K.M.T. performed the data analysis and interpretation under the supervision of M.K.N. and R.C.O. C.B.C. drafted the paper, and all authors provided critical revisions. All authors approved the final version of the paper for submission. The authors also wish to acknowledge Britta Ekdahl, Julia Harris, Amy Harmon, Sara Slama, and Catherine Glenn, Rebecca Forrester, and Julie Drummond for their assistance with data collection and data management, as well as Katherine DiVasto, Ilana Gratch, and Seoho Hahm, for their assistance reviewing this manuscript. This research was supported by the Sackler Scholar Award in Psychobiology and the Harvard Merit/Term Time Research Award (Site 1). None of these funding sources represent conflicts of interest. 
Table 1. Demographic, clinical, and contextual correlates of Death/Life IAT and Suicide Stroop performance

\begin{tabular}{|c|c|c|c|c|}
\hline & $\mathbf{I A T}_{\text {pre }}$ & IAT $_{\text {post }}$ & Strooppre & Strooppost \\
\hline \multicolumn{5}{|l|}{ Demographics } \\
\hline Age & $-.28 * * *$ & $-.25 * * *$ & $.14^{*}$ & .05 \\
\hline Gender $^{\mathrm{a}}$ & .07 & -.08 & -.15 & .08 \\
\hline \multicolumn{5}{|l|}{ Clinical Severity } \\
\hline Depressive Symptoms & $.25 * * *$ & $.34 * * *$ & -.01 & .12 \\
\hline \multicolumn{5}{|l|}{ Mood Induction } \\
\hline Valence $_{\text {pre }}$ & $-.21 * *$ & $-.20 * *$ & -.03 & -.09 \\
\hline Valence $_{\text {post }}$ & $-.22 * * *$ & $-.21 * *$ & -.03 & -.07 \\
\hline Arousal $_{\text {pre }}$ & -.10 & -.04 & .06 & -.02 \\
\hline Arousal $_{\text {post }}$ & .06 & -.01 & -.00 & -.02 \\
\hline
\end{tabular}

Note. Pearson's $r$ values represented above, with the exception of gender which features Cohen's $d$ values. IAT=Implicit Association Test/ Pre and Post refer to time points immediately before and after the negative mood induction. Mood valence and arousal ratings represented via z scores. Depressive Symptoms were measured via Beck Depression Inventory.

$* p<.05 \quad * * p<.01 \quad * * * p<.001$

${ }^{a}$ Cohen's $d$ values. 
Table 2. IAT performance predicting future suicidal ideation

\begin{tabular}{|c|c|c|c|c|c|}
\hline & & \multicolumn{4}{|c|}{ 1-month follow-up } \\
\hline & & \multicolumn{2}{|c|}{ Bivariate } & \multicolumn{2}{|c|}{ Multivariate } \\
\hline & & OR & $\mathrm{CI}$ & OR & $\mathrm{CI}$ \\
\hline \multirow[t]{2}{*}{ Cont. } & Pre & $3.20 * *$ & $1.53-6.68$ & 2.41 & $0.98-5.92$ \\
\hline & Post & $3.38 * * *$ & $1.71-6.70$ & 1.88 & $0.83-4.22$ \\
\hline \multirow[t]{5}{*}{ Cat. } & Pre & $4.56 * * *$ & $2.07-10.05$ & $3.38 *$ & $1.26-9.10$ \\
\hline & Post & $3.86 * * *$ & $1.89-7.85$ & $2.80 *$ & $1.19-6.57$ \\
\hline & & \multicolumn{4}{|c|}{ 6-month follow-up } \\
\hline & & \multicolumn{2}{|c|}{ Bivariate } & \multicolumn{2}{|c|}{ Multivariate } \\
\hline & & OR & CI & OR & $\mathrm{CI}$ \\
\hline \multirow[t]{2}{*}{ Cont. } & Pre & $2.88 * *$ & $1.45-5.74$ & 1.76 & $0.68-4.59$ \\
\hline & Post & $4.30 * * *$ & $2.15-8.60$ & 2.27 & $0.94-5.51$ \\
\hline \multirow[t]{2}{*}{ Cat. } & Pre & $2.96 * *$ & $1.33-6.56$ & 1.69 & $0.58-4.91$ \\
\hline & Post & $4.47 * * *$ & $2.07-9.62$ & $2.77 *$ & $1.04-7.40$ \\
\hline
\end{tabular}

Note. IAT=Death/Life Implicit Association Test; SI=Suicidal Ideation; Cont.=Continuous IAT scores; Cat.=Dichotomous IAT scores. Multivariate model controlled for site type (Site 1 vs. Site 2), age, gender, depressive symptoms, pre-/post-induction mood valence ratings, as well as baseline (past year) frequency of suicidal ideation.

$* p<.05, * * p<.01$ 
Figure 1. Baseline Group Differences in Death/Life IAT and Stroop Performance

a. Death/Life IAT Performance

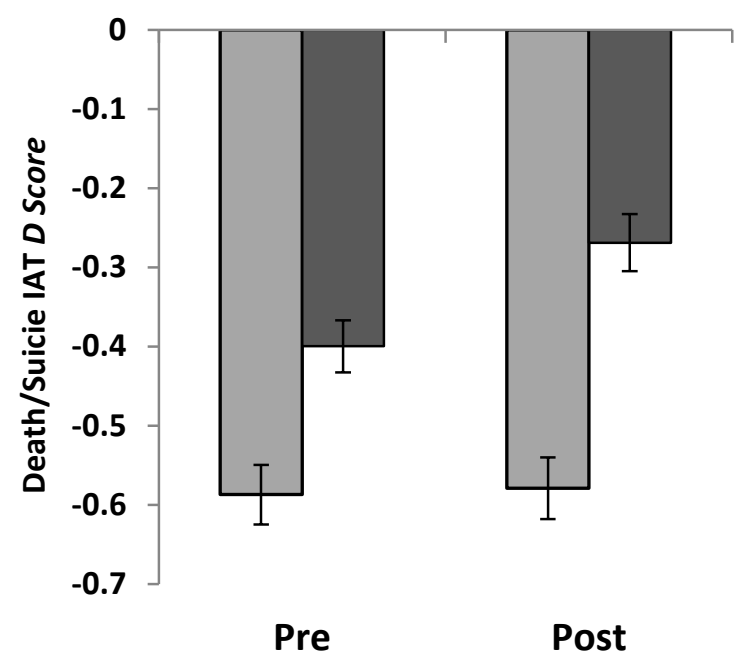

b. Suicide Stroop Performance

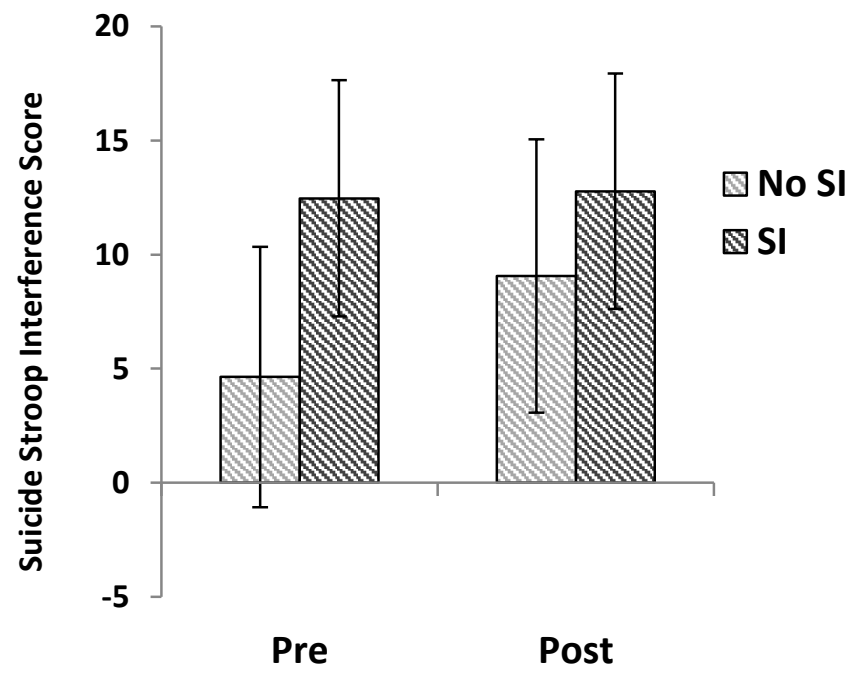

Note. IAT=Death/Life Implicit Association Test; SI=suicidal ideation; ms=millisecond; Pre=Prenegative mood induction; Post=Post-negative mood induction. Suicide ideator performance marked in dark grey; non-ideator performance marked in light grey. Scores indicate average continuous performance scores. Higher IAT scores indicate weaker implicit association between Me-Life ( $\mathrm{Not} \mathrm{Me-}$ Life). Higher Stroop scores indicate poorer attentional bias in the context of death-related words. 
Click here to access/download Supplemental Material - Integral IATstroop_suppmaterials_CLEAN_032818.docx 